\section{„Keine Untersuchungen vorenthalten“}

Aufgrund umfangreicher Sicherheitsmaßnahmen und Anwendungseinschränkungen ist die Anzahl neu aufgetretener Fälle von Nephrogener Systemischer Fibrose (NSF) nach der Gabe gadoliniumhaltiger Kontrastmittel nahezu 0. Doch die Furcht vor NSF sorgt weiterhin für Verwirrung, sagt Professor Dr. Johannes T. Heverhagen, Chefarzt für Radiologie am Inselspital Bern (CH). Der Arzt und Physiker hat gemeinsam mit Frau Prof. Gabriele Krombach (Gießen) und Frau Prof. Elke Gizewski (Innsbruck, AT) eine Handreichung für die Gabe dieser Kontrastmittel verfasst.

Ist die Nephrogene Systemische Fibrose aktuell noch ein Thema, wenn es um die Sicherheit von MRT-Kontrastmittel geht?

Heverhagen: Das Thema ist weiterhin sehr groß und wichtig, da Unsicherheiten bezüglich der Risikofaktoren immer noch da sind. Teilweise gibt es von Klinik zu Klinik unterschiedliche Regelungen, was zu Verwirrungen führt.

\section{? Welche Fehler werden aus Ihrer Sicht gemacht?}

Heverhagen: Ein häufiger Fehler ist es, Patienten mit bekannter Niereninsuffizienz die kontrastmittelgestützte MR-Untersuchung vorzuenthalten oder die Untersuchung nativ durchzuführen - mit den daraus resultierenden diagnostischen Schwächen. Wir wissen aber heute, dass eine Niereninsuffizienz keine Kontraindikation für die Gabe von gadolinumhaltigen Kontrastmitteln bedeutet, sondern die Wahl des Präparats und die Dosierung abgewogen werden muss.

\section{Worauf sollten die Kollegen bei der Gabe von MR-Kontrastmitteln ach- ten?}

Heverhagen: Es sind aktuell 8 Kontrastmittelpräparate auf dem Markt, die auf Basis von Gadolinium wirken. 3 davon sind stark mit NSF assoziiert und sollten nur nierengesunden Patienten verabreicht werden, 5 gehören nicht in die Hoch-Risikogruppe und dürfen auch für die Diagnostik bei nierengeschädigten Patienten eingesetzt werden. Immer gilt der Hinweis auf die geringst mögliche Dosierung. Gerade das stärker konzentrierte Kontrastmittel (Gadovist) kann zu einer höheren Dosierung verleiten. Kontrastmittel mit teilweise biliärer Ausscheidung (Primovist und MultiHance) könnten hier protektiv wirken. Alles in allem gilt: Die kontrastmittelverstärkte MRT stellt trotz NSF-Risikos ein sicheres diagnostisches Verfahren dar, das bei Beachtung der Anwendungsrichtlinien und aktuellen Empfehlungen auch bei Patienten mit schwerer Niereninsuffizienz sicher eingesetzt werden kann und nicht zwingend durch andere Verfahren ersetzt werden muss.

Vielen Dank für das Gespräch!

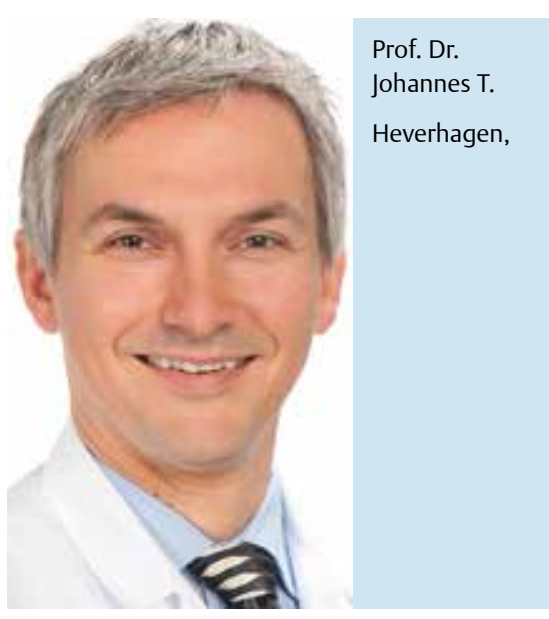

Die Arbeit von Prof. Heverhagen et. al. Application of Extracellular Gadoliniumbased MRI Contrast Agents and the Risk of Nephrogenic Systemic Fibrosis erschien im Juli 2014 in der RöFo - Fortschritte auf dem Gebiet der Röntgenstrahlen: https:// www.thieme-connect.com/products/ ejournals/ abstract/10.1055/s-0033-1356403 